\title{
Review of: "Revealing a hidden intermediate of rotatory catalysis with X-ray crystallography and Molecular simulations"
}

ichiro yamato ${ }^{1}$

1 Tokyo University of Science

Potential competing interests: The author(s) declared that no potential competing interests exist.

A typical rotatory bio-motor protein, E. hirae V1-ATPase, has been studied crystallographically and computationally. It is composed of three identical A1B1 subunits and a central stalk DF complex. Interface between each A1B1 has one ATP binding site and its hydrolysis drives the DF rotation. Within the rotation cycle of the ATPase, three dwell states, catalytic, ATP-binding, and ADP-release dwells, have been shown. In this new report, a new dwell state, Pi-release dwell, after catalytic and before ATP-binding dwell, is identified as Al-F4 inhibited ATPase crystals. Using molecular dynamics simulation, the Pi-release path through the subunit interface is proposed, which seems to induce the long distance conformational change through subunit interfaces and finally to produce the next dwell state, ATP-binding dwell. Furthermore, the elastic nature of the DF stalk seems essential for the Pi-release and the conformational changes. Therefore, this report adds one step further insight into the chemomechanical coupling mechanism of bio-energy transducing machineries. 